\title{
Joint crisis plans for people with psychosis: economic evaluation of a randomised controlled trial
}

\author{
Chris Flood, Sarah Byford, Claire Henderson, Morven Leese, Graham Thornicroft, Kim Sutherby, George Szmukler
}

\begin{abstract}
Objective To investigate the cost effectiveness of joint crisis plans, a form of advance agreement for people with severe mental illness.

Design Single blind randomised controlled trial.

Setting Eight community mental health teams in southern England.

Participants 160 people with a diagnosis of psychotic illness or non-psychotic bipolar disorder who had been admitted to hospital at least once within the previous two years.

Intervention Joint crisis plan formulated by the patient, care coordinator, psychiatrist, and project worker containing contact information, details of illnesses, treatments, relapse indicators, and advance statements of preferences for care for future relapses. Control group was standardised service information. Main outcome measures Admission to hospital; service use over 15 months.

Results Use of a joint crisis plan was associated with less service use and lower costs on average than in the standardised service information group, but differences were not significant. Total costs during follow-up were $£ 7264$ (€10 616, \$13 560) for each participant with a joint crisis plan and $£ 8359$ (€12 217, $\$ 15609)$ for each participant with standardised service information (mean difference $£ 1095$; $95 \%$ confidence interval -2814 to 5004). Cost effectiveness acceptability curves, used to explore uncertainty in estimates of costs and effects, suggest there is a greater than $78 \%$ probability that joint crisis plans are more cost effective than standardised service information in reducing the proportion of patients admitted to hospital. Conclusion Joint crisis plans produced a non-significant decrease in admissions and total costs. Though the cost estimates had wide confidence intervals, the associated uncertainty suggests there is a relatively high probability of the plans being more cost effective than standardised service information for people with psychotic disorders.
\end{abstract}

\section{Introduction}

Many people with a diagnosis of a psychotic disorder have high rates of relapse, which may result in numerous admissions to inpatient psychiatric units. This can be distressing for the individuals concerned as well as costly for society. In 1998 Patel and Knapp calculated the annual cost to the UK National Health Service (NHS) of inpatient and residential provision for schizophrenia and other non-organic psychoses as $£ 946 \mathrm{~m}$ $(€ 1382 \mathrm{~m}, \$ 1766 \mathrm{~m}) .{ }^{1}$ Morris et al and Rice and Miller showed that $79 \%$ of direct costs are incurred by only $10 \%$ of patients. ${ }^{23}$
The rationale for the joint crisis plan has been previously reported. ${ }^{4}$ Briefly, these plans aim to mitigate some of the negative consequences of relapse, including admission to hospital, use of coercion in the form of the Mental Health Act, and associated costs. The plan establishes the preferences for treatment of those who use the service at a point when they are relatively well, to be applied in any subsequent crisis when the individual may be too unwell to indicate their preferences. The aim is to reach agreement between the service user and clinical team through negotiation and consensus building, facilitated by a third party.

A randomised controlled trial comparing joint crisis plans with a control intervention in which patients were supplied with information leaflets relevant to their mental health care (standardised service information) showed fewer admissions at a borderline level of significance and significantly reduced use of the Mental Health Act in the joint crisis plan group. ${ }^{4}$ We are reporting the results of the economic evaluation for this trial.

\section{Methods}

Our primary hypothesis was that joint crisis plans in addition to usual care will be more cost effective than standardised service information in addition to usual care because of reductions in admissions to hospital and total treatment costs.

The methods of the trial have been reported elsewhere. ${ }^{4}$ Participants were recruited from seven different community mental health teams across London and one in Kent. Eligible patients had a clinical diagnosis of psychotic illness, were not currently receiving inpatient care, and had experienced an admission in the previous two years.

\section{Interventions}

A facilitator, an experienced mental health professional external to the teams participating in the trial and not involved in the participants' care, coordinated the development of a joint crisis plan. At an introductory meeting the facilitator explained the procedure to the participating service user and care coordinator and discussed possible contents of the plan. The facilitator finalised the contents at a second meeting with the service user, care coordinator, and psychiatrist and included contact information, details of mental and physical illness and their treatment, early indicators of relapse, and advance statements of preferences for care in the event of relapse. People specified by the service user, typically key local services and named friends and family members, received a copy of the plan.

Members of the standardised service information control group received information leaflets on different types of mental illness and medication, local services, the Mental Health Act, 
complaints procedures, and access to case records, in addition to receiving treatment as usual.

\section{Outcomes}

We assessed outcomes at 15 months after trial entry. These included admission to hospital, length of time spent in hospital (primary outcome measures ${ }^{4}$ ), and objective coercion (compulsory treatment under the Mental Health Act 1983).

\section{Costs}

We assessed costs from the perspective of service providers, including the NHS, social services, and the criminal justice system. We also considered the perspectives of the user and carer in terms of out of pocket expenses. We collected data on use of services from interviews with patients using a modified version of the client service receipt inventory. ${ }^{6}$ This was supplemented by data on hospital admission, bed days, and use of the Mental Health Act from computerised hospital clinical activity systems and Mental Health Act office records.

All costs were calculated for the 2000-1 financial year. Because of lack of data on the timing of resources used, we could not discount costs that occurred after the first 12 months of the trial. Given that the data covered 15 months, it is unlikely that the lack of discounting for costs occurring over a three month period would significantly affect the results reported.

We costed the joint crisis plan on the basis of the time spent by the facilitator and clinical teams in producing the crisis plans, plus relevant administrative, managerial, and capital overheads. The cost of the control group intervention was calculated on the basis of the actual purchase cost of the information leaflets with the cost of the facilitator's time in distributing them.

We derived unit costs from published cost templates ${ }^{7}$ to cost the administration of the Mental Health Act, including initial assessment, managers' hearings and tribunal hearings, report preparation, legal aid provision, and administration. We costed secondary healthcare services using NHS reference $\operatorname{costs}^{8}$ and information provided by the South London and Maudsley NHS Trust finance department. Published unit costs were applied to community health and social services, ${ }^{9}$ prison stays,${ }^{10}$ medications, ${ }^{11}$ and criminal justice resources (contact with lawyers, probation and police officers, arrests, court appearances, and nights in police cells). ${ }^{7}$

As most people with psychotic disorders are unemployed, changes in productivity are unlikely to be relevant so we did not measure losses of productivity as a result of time off work due to illness.

\section{Statistical analyses}

Statistical analyses were carried out on an intention to treat basis. Unit costs were applied to individual service use data and aggregated. Despite the skewed nature of cost data, we analysed differences in costs between the two groups using the standard $t$ test and confirmed the validity of results using bootstrapping. ${ }^{12} \mathrm{We}$ have not reported the bootstrapped results as the confidence intervals were similar to $t$ test results in all tests. The advantage of this approach is the ability to make inferences about the arithmetic mean. ${ }^{13}$

We assessed cost effectiveness by calculating incremental cost effectiveness ratios, ${ }^{14}{ }^{15}$ which measure the difference in average costs between the two trial arms divided by the difference in average effects. In the primary analysis we explored cost effectiveness in terms of admissions, the primary outcome of the clinical trial. While it would have been possible to choose number of admissions or number of days in hospital, both these variables were associated with relatively high proportions of zero responses. Instead, we selected the proportion of patients admitted over the follow-up period as the main measure of outcome. Comparison of the results using the three different variables showed similar results, thus supporting the validity of this choice. In secondary analyses we explored cost effectiveness in terms of use of the Mental Health Act (proportion of patients experiencing compulsory detention over the follow-up period). However, the results did not differ from the primary analyses and so we have not reported them here.

We used repeat re-sampling from the costs and effectiveness data using non-parametric bootstrapping to generate a distribution of mean costs and effects for the two treatments. ${ }^{12}$ These distributions were used to plot cost effectiveness acceptability curves, which show the probability that the joint crisis plan is cost effective compared with standardised service information for a range of maximum monetary values (ceiling ratios, $\lambda$ ) that a decision maker might be willing to pay for a reduction in the proportion of patients admitted to hospital. ${ }^{16}$ These acceptability curves illustrate the uncertainty associated with the estimate of costs and effects as a result of sampling variation and were developed as a way of overcoming the statistical difficulties of calculating confidence intervals around incremental cost effectiveness ratios. ${ }^{17}{ }^{18}$ We performed several one way sensitivity analyses to ascertain thresholds for cost effectiveness and to explore the robustness of results to changes in the cost data.

\section{Results}

\section{Patients}

We assessed 466 patients for eligibility. Twenty three did not meet the criteria for inclusion. Of the remainder, 283 patients were either not contacted or contacted and declined to take part. The 160 patients remaining were individually randomised to each arm, and we collected the economic data over a period of 15 months.

\section{Outcomes}

Use of the Mental Health Act was significantly reduced in the joint crisis plan group, with compulsory admission experienced by $13 \%$ compared with $27 \%$ in the standardised service information group (risk ratio $0.48,95 \%$ confidence interval 0.24 to 0.95 , $\mathrm{P}=0.03$ ). There were also fewer admissions to hospital in the joint crisis plan group, but this difference was not significant ( $30 \%$ v $44 \%$; ratio $0.69,0.45$ to $1.04, \mathrm{P}=0.07$ ).

\section{Service use}

Table 1 shows the mean number of contacts that patients had with each service over the 15 month follow-up. Patients with joint crisis plans had fewer psychiatric admissions and outpatient and accident and emergency attendances, Mental Health Act episodes, attendances at a day centre, contacts with social workers, and criminal justice contacts. The control group had fewer non-psychiatric admissions to hospital, fewer contacts with general practitioners and community psychiatric nurses, and used less specialised accommodation. There were no differences in the receipt of social security benefits or out of pocket expenses between the two groups.

\section{Costs}

The mean total cost per patient over the 15 month study period was lower in the joint crisis plan group than in the control group (£7264 (€10 616, \$13 560) v £8359 (€12 217, \$15 609), respectively), but this difference was not significant (mean difference $£ 1095 ; 95 \%$ confidence interval -2814 to $5004 ; \mathrm{P}=0.57$ ) (table 2). Nor were there any significant differences in cost by providing sector. 
Table 1 Mean (SD) use of resources over the 15 month follow-up period

\begin{tabular}{|c|c|c|}
\hline Service & Joint crisis plan $(\mathrm{n}=\mathbf{8 0})$ & $\begin{array}{l}\text { Standardised service } \\
\text { information }(n=80)\end{array}$ \\
\hline \multicolumn{3}{|l|}{ Mental health services } \\
\hline Psychiatric inpatient days & $32.0(73.0)$ & $36.0(69.0)$ \\
\hline Psychiatric outpatient attendances & $1.5(2.0)$ & $1.6(2.0)$ \\
\hline $\begin{array}{l}\text { Community psychiatric nursing } \\
\text { contacts }\end{array}$ & $185.0(292.0)$ & $170.0(292.0)$ \\
\hline Psychiatric day service contacts & $1.0(9.0)$ & $1.5(12.0)$ \\
\hline $\begin{array}{l}\text { Other community day centre } \\
\text { contacts }\end{array}$ & $5.0(25.0)$ & $13.0(38.0)$ \\
\hline Mental health act assessments & $0.1(0.4)$ & $0.3(0.4)$ \\
\hline \multicolumn{3}{|l|}{ Other health services } \\
\hline Non-psychiatric inpatient days & $18.0(26.0)$ & $2.5(2.0)$ \\
\hline $\begin{array}{l}\text { Non-psychiatric outpatient } \\
\text { attendances }\end{array}$ & $18.0(47.0)$ & $33.0(89.0)$ \\
\hline $\begin{array}{l}\text { Accident and emergency contacts } \\
\text { (non-psychiatric) }\end{array}$ & $0.1(0.2)$ & $0.2(1.0)$ \\
\hline $\begin{array}{l}\text { Accident and emergency contacts } \\
\text { (psychiatric) }\end{array}$ & $0.1(0.4)$ & $0.2(0.5)$ \\
\hline General practitioner contacts & $1.9(4.0)$ & $1.3(2.0)$ \\
\hline $\begin{array}{l}\text { No of different medications } \\
\text { prescribed }\end{array}$ & $1.9(1.8)$ & $1.7(1.4)$ \\
\hline \multicolumn{3}{|l|}{ Social services } \\
\hline Social work hours & $32.0(74.0)$ & $138.0(217.0)$ \\
\hline Specialised accommodation weeks & $0.3(0.4)$ & $0.2(0.4)$ \\
\hline \multicolumn{3}{|l|}{ Criminal justice } \\
\hline Arrests & $0.1(0.4)$ & $0.2(0.9)$ \\
\hline Contacts with lawyer & $0.3(1.3)$ & $0.3(1.6)$ \\
\hline Court appearances & $0.0(0.2)$ & $0.1(0.7)$ \\
\hline Contacts with probation officer & 0 & 0 \\
\hline Contacts with police officer & $0.5(1.2)$ & $0.7(1.5)$ \\
\hline Nights in police cell & $0.1(0.3)$ & $0.1(0.4)$ \\
\hline Nights in prison & $0.2(1.4)$ & $6.8(46.0)$ \\
\hline
\end{tabular}

\section{Sensitivity analysis}

Threshold analysis revealed that, on average, an additional reduction of 14 days in hospital per patient in the joint crisis plan group would be required before the difference in mean total cost per patient between the two groups became significant (mean difference $£ 3381,95 \%$ confidence interval 27 to $6735, \mathrm{P}=0.05$ ). We varied unit costs for bed days, the key cost in this sample, to explore their impact on the reported results. There was no impact on the significance of the difference in mean total cost per patient even when the cost of bed days was halved ( $£ 799$, -1308 to $2906, \mathrm{P}=0.46$ ) or doubled (mean difference $£ 1688$, -5900 to $9274, \mathrm{P}=0.66$ ). Increasing the cost of the joint crisis plan intervention to bias the results against the intervention, even with a fourfold increase in facilitator time, did not alter the

Table 2 Mean (SD) total cost (£) per patient over the 15 month follow-up period, 2000-1 prices

\begin{tabular}{lccc} 
Service & $\begin{array}{c}\text { Joint crisis plan } \\
(\mathbf{n}=\mathbf{8 0})\end{array}$ & $\begin{array}{c}\text { Standardised service } \\
\text { information } \mathbf{( n = 8 0 )}\end{array}$ & $\begin{array}{c}\text { Mean difference (95\% } \\
\mathbf{C I})\end{array}$ \\
\hline Mental health services: & & & \\
\hline Total cost & $6064(12638)$ & $6912(12022)$ & $875(-2948$ to 4698$)$ \\
\hline JCP/SSI & $44(28)$ & $18(3)$ & $-26(-32$ to -20$)$ \\
\hline Other health services & $1016(1343)$ & $1033(1006)$ & $17(-350$ to 385) \\
\hline Social services & $116(187)$ & $110(229)$ & $-6(-71$ to 59$)$ \\
\hline Criminal justice sector & $37(138)$ & $274(1666)$ & $238(-129$ to 604$)$ \\
\hline Out of pocket expenses: & & & $-6(-15$ to 3$)$ \\
\hline Patient & $14(36)$ & $7(20)$ & $4(-22$ to 29$)$ \\
\hline Carer & $18(61)$ & $22(99)$ & $1095(-2814 \text { to } 5004)^{\star}$ \\
\hline Total costs & $7264(13045)$ & $8359(12168)$ &
\end{tabular}

JCP=joint crisis plan; $\mathrm{SSI}=$ standardised service information. ${ }^{*} \mathrm{P}=0.58$.

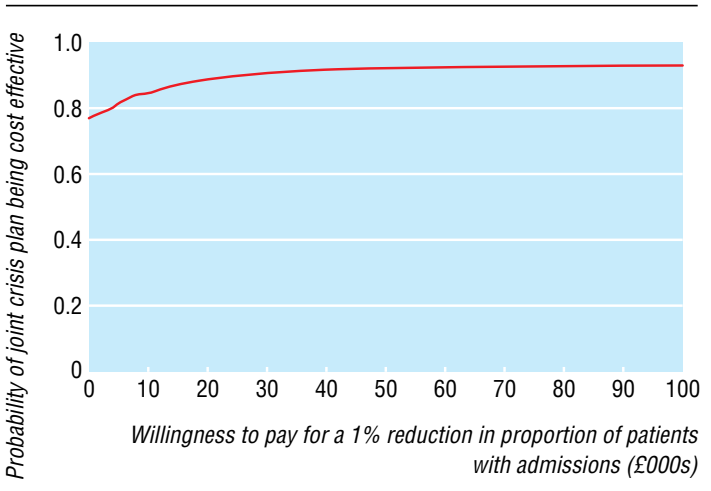

Fig 1 Cost effectiveness acceptability curve for admissions over 15 month follow-up period

significance of the cost comparison (£963, - 2944 to 4869 , $\mathrm{P}=0.63$ )

\section{Cost effectiveness analysis}

The incremental cost effectiveness ratio for admissions over the 15 month follow-up period was - $£ 131$ per $1 \%$ reduction in the proportion of patients admitted to hospital. Observed differences show that the joint crisis plan was cheaper and more effective on average than standardised service information. Figure 1 shows the cost effectiveness acceptability curve associated with the proportion of admissions. This shows that the joint crisis plan is superior to standardised service information over the full range of values of the ceiling ratio plotted. The curve for the joint crisis plan shows that there is at least a $78 \%$ probability that the plan is more cost effective than standardised service information in preventing admissions (range $78 \%$ to $94 \%$ ). Figure 2 shows the distribution of the bootstrap replicates used to create the cost effectiveness acceptability curves and illustrates the extent of the uncertainty. Because a higher effectiveness score is undesirable ( $1=$ admission, $0=$ no admission), moving from left to right on the $\mathrm{x}$ axis means a worsening in the incremental effectiveness for the joint crisis plan group compared with the control group. This means that, contrary to conventional economic cost effectiveness plans, the south west quadrant contains those points where the experimental intervention is superior to the control intervention-that is, costs less and is more effective. The scatter plot clearly shows that most points lie within the desired south west quadrant, showing strong evidence of differences in costs and effects in favour of the joint crisis plan group (fig 2).

We re-ran the cost effectiveness acceptability curve analysis using the cost data from the four sensitivity analyses, described

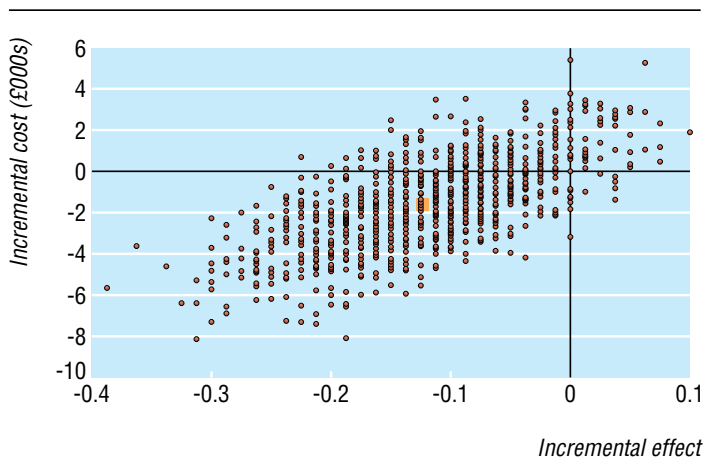

Fig 2 Distribution of bootstrap replicates used to create cost effectiveness acceptability curve (the square represents the incremental cost effectiveness ratio) 


\section{Research}

above, to further explore the effect of such variations. The probability that joint crisis plans are more cost effective than standardised service information fell slightly in the analyses biased against the joint crisis plan intervention but did not fall below $67 \%$ at any point. Increasing facilitator time, and thus costs, showed the greatest variability with the probability of the joint crisis plan group being more cost effective than the control ranging from $67 \%$ to $95 \%$.

\section{Discussion}

Crisis planning interventions seem to be a cost effective method of preventing admissions and compulsory treatment for people with psychotic disorders. In this study there were fewer compulsory admissions in the joint crisis plan group than the standardised service information group. Numbers of total admissions and total costs were also lower in the plan group, but these differences were not significant. Exploration of the uncertainty surrounding the estimates of costs and effects suggests that there is at least a $78 \%$ probability that joint crisis plans are a more cost effective strategy than standardised service information for reducing the probability of admissions, irrespective of the amount a decision maker may be willing to pay for such reductions. Attempts to bias against the intervention in sensitivity analysis reduced this probability somewhat, but there was still a minimum probability in favour of joint crisis plans of $67 \%$.

In the long term, joint crisis plans are not particularly resource intensive; the marginal cost of facilitating an additional joint crisis plan is relatively small. In the short term, however, the provision of facilitated plans may involve investment in the salaries of the facilitators. Whether this is affordable must be considered with evidence of the probable cost effectiveness of joint crisis plans and any savings made by reducing admissions.

One limitation of the current study was the lack of a measure of change from the patients' perspective. It is reasonable to assume, however, that fewer admissions, particularly compulsory admissions, would have a positive impact on quality of life. Such perspectives should be explored in future research of advance statements.

Our findings are of interest with respect to a new or amended Mental Health Act in England. A parliamentary committee has commented that measures proposed by the government could erode civil liberties and be too heavily focused on compulsion. ${ }^{19}$ Advance statements in the form of joint crisis plans may have the potential to reduce both compulsion and costs.

Contributors: $\mathrm{CF}$ and $\mathrm{SB}$ undertook the economic evaluation and wrote the paper. CF, CH, ML, GT, KS, and GS all took part in the project and contributed to writing the paper. GT is guarantor.

Funding: $\mathrm{CH}$ was funded by a Medical Research Council health service research training fellowship, and CF was funded by Bethlem and Maudsley research and development support.

Competing interest declaration: None declared.

Ethical approval: Ethics committees of the South London and Maudsley NHS Trust, Lewisham University Hospital, South West London and St George's NHS Trust, and Thames Gateway NHS Trust.

1 Patel A, Knapp M. Costs of mental illness in England. Mental Health Res Rev 1998;5:4 10 .

2 Morris S, Hogan T, McGuire A. The cost-effectiveness of clozapine. A survey of the literature. Clin Pharmacoeconomics 1998;15:137-52.

3 Rice D, Miller L. The economic burden of schizophrenia: conceptual and methodological issues, and cost estimates. In: Moscarelli M, Rupp A, Sartorius N, eds. Handbook of mental health economics and health policy. Vol 1. Schizophrenia. Chichester: John Wiley, 1996.

4 Henderson C, Flood C, Leese M, Thornicroft G, Sutherby K, Szmukler G. Effect of joint crisis plans on use of compulsory treatment in psychiatry: single blind randomised controlled trial. BMJ 2004;329:136-8.
5 Sutherby K, Szmukler GI, Halpern A, Alexander M, Thornicroft G, Johnson C, et al. A study of "crisis cards" in a community psychiatric service. Acta Psychiatr Scand 1999;100:56-61.

6 Beecham J, Knapp M. Costing psychiatric interventions. In: Thornicroft G, ed. Measur ing mental health needs. London: Royal College of Physicians/Gaskell, 2001:200-24

7 Knapp M, Chisholm D, McCrone P, Almond S, Amponsah S. The resource implications of the Mental Health Act. London: London School of Economics and Institute of Psychiatry, Kings College London, 1999.

8 Department of Health. NHS reference costs. London: DoH, 2001.

9 Netten A, Curtis L. Unit costs of health and social care. Canterbury: Personal Social Services Research Unit, University of Kent, 2000.

10 Prison Service. Prison service annual report and accounts. London: Stationery Office, 2000.

11 British Medical Association, Royal Pharmaceutical Society of Great Britain. British national formulary. London: BMA, RPS, 2001. (No 42.)

12 Efron B, Tibshirani R. An introduction to the bootstrap. New York: Chapman and Hall, 1993.

13 Barber JA, Thompson SG. Analysis of cost data in randomized trials: an application of the non-parametric bootstrap. Stat Med 2000;19:3219-36.

14 Van Hout B, Al M, Gordon G. Costs, effects and c/e-ratios alongside a clinical trial Health Econ 1994;3:309-19.

15 Briggs A. Handling uncertainty in economic evaluation and presenting the results. In: Drummond M, McGuire A, eds. Economic evaluation in health care: merging theory and Drummond M, McGuire A, eds. Economic evaluation in health care: me

16 Fenwick E, Claxton K, Sculpher M. Representing uncertainty: the role of Fenwick E, Claxton K, Sculpher M. Representing uncertainty
cost-effectiveness acceptability curves. Health Econ 2001;10:779-87.

17 Fenwick E, Byford S. A guide to cost-effectiveness acceptability curves. Br J Psychiatry 2005; $187: 106-8$

18 Fenwick E, O'Brien BJ, Briggs A. Cost-effectiveness acceptability curves-facts, fallacies and frequently asked questions. Health Econ 2004;13:405-15.

19 House of Lords House of Commons Joint Committee. Draft Mental Health Bill. Vol 1. London: Stationery Office, 2005. (HL Paper 79-1 HH.)

(Accepted 19 July 2006)

doi $10.1136 /$ bmj. 38929.653704 .55

Department of Mental Health and Learning Disability, City University, London E1 2EA

Chris Flood lecturer in mental health

Centre for the Economics of Mental Health, Institute of Psychiatry, King's College, London SE5 8AF

Sarah Byford senior lecturer in health economics

Section of Community Psychiatry, Health Service Research Department, Institute of Psychiatry, King's College, London SE5 8AF

Claire Henderson MRC training fellow in health services research

Health Service Research Department, Institute of Psychiatry, King's College, London SE5 8AF

Morven Leese medical statistician

Graham Thornicroft professor of community psychiatry

South London and Maudsley NHS Trust, Croydon CR0 1XT

Kim Sutherby consultant psychiatrist

Institute of Psychiatry, King's College, London

George Szmukler dean

\section{What is already known on this topic}

Joint crisis plans, formulated jointly by a mental health service user, a clinician, and a facilitator, are highly acceptable to service users

Such plans have been shown to approximately halve the use of compulsion during psychiatric hospital admission for people with psychotic disorders

People with psychotic disorders who create and use plans may also have modestly reduced hospital admission rates

\section{What this study adds}

Over 15 months total costs were $£ 7264$ for each person with a joint crisis plan and $£ 8359$ for each person with standardised service information

Cost effectiveness acceptability curves suggest there is a greater than $78 \%$ probability that joint crisis plans are more cost effective than standardised service information in reducing the proportion of patients admitted to hospital 
Research

Correspondence to: S Byford s.byford@iop.kcl.ac.uk 